\title{
3 Zentrale Fragestellungen der Studie
}

Während türkische, arabische oder chinesische Geschäfte bzw. Unternehmen im Stadtraum häufig gut sichtbar verortet sind, existiert die ethnische Ökonomie der Kurd/inn/en in Wien zumeist im Verborgenen. Zudem weist die in Wien lebende kurdische Diaspora eine äußerst heterogene Struktur auf. Es bestehen Unterschiede hinsichtlich der nationalen und geographischen Herkunft, der sozialen Zugehörigkeit, politischer und konfessioneller Orientierungen etc. Am wenigsten ist über die kleineren Gruppen von Kurd/inn/en, die nicht aus der Türkei, sondern aus dem Irak, dem Iran oder Syrien zugewandert sind, bekannt. Die zahlenmäßige Größe der kurdischen Diaspora in Wien kann nur geschätzt werden. Noch weniger existieren einigermaßen verlässliche Daten zur Rolle und Struktur der Diaspora-Entrepreneurship der Kurd/inn/en in Wien und schon gar nicht zur Relevanz selbständiger Erwerbstätigkeit für das Gender Empowerment kurdischer Frauen.

Bei Frauen spielt die Involvierung in selbständige Erwerbstätigkeit für ihr Empowerment in der eigenen Gruppe eine wichtige Rolle. Die traditionelle Rolle der kurdischen Frau unterscheidet sich zwar nicht grundlegend von jener in anderen Gruppen im Mittleren Osten (SUAD \& AFSĀNEH 2003), bedingt durch politische Aktivistinnen, Frauen im organisierten Widerstand sowie vielfältige Wechselwirkungen zwischen Migration und politischer Mobilisierung hat sich aber in den letzten Jahrzehnten eine sehr aktive kurdische Frauenbewegung konstituiert ${ }^{7}$ (AzIZOĞLU-BAZAN 2017). In der kurdischen Diaspora spiegelt sich dies darin wider, dass Frauen auch als Unternehmerinnen aktiv sind und nicht nur als in Familienunternehmen Tätige. Für Wien sind allerdings ,hard facts“ dazu bislang nicht erhoben worden.

Gerade im Falle der kurdischen Diaspora sind auch politische Aspekte der Community-Identität, die im Immigrationskontext entwickelt werden, relevant (vgl. CLIFFORD 1994). In Demokratien westlicher Prägung profitieren Diasporas von ihrer Anerkennung. Nicht nur der nationale Kontext im Migrationszielland, sondern gerade auch der lokale politische Rahmen in der Stadt, in der die Diaspora-Community sowie die Diaspora-Unternehmer/innen lokalisiert sind, spielt eine Rolle (VERTOVEC 2005). Aufgrund der Vielfalt an Verflechtungen ist daher für eine Erhebung der Fokus auf den abgegrenzten räumlichen Kontext der Diaspora in einer Stadt wie Wien am erfolgversprechendsten. Die diversitätsorientierte Integrationspolitik der Stadt Wien ${ }^{8}$

$7 \quad$ Als zentraler Bestandteil der Freiheitsbewegung will die kurdische Frauenbewegung ein alternatives, demokratisches Gesellschaftssystem erschaffen. Die Befreiung der Frauen, Gleichheit, Zusammenarbeit der verschiedenen Volks- und Religionsgemeinschaften stehen im Vordergrund, vgl. <https://civaka-azad.org/tag/kurdische-frauenbewegung/>.

$8 \quad$ Vgl. $<$ https://www.wien.gv.at/menschen/integration/pdf/integrationsleitlinien.pdf $>$. 
gestattet im besonderen Ausmaß die Artikulation kurdischer ethnischer Identitäten, den Protest gegen politische Verfolgung und Diskriminierung in den Herkunftsländern und damit einhergehende politische Forderungen. Eine vielfach genutzte Möglichkeit dieser Artikulation stellen kulturelle Angebote für die Bevölkerung der Aufnahmegesellschaft sowie kurdische Diaspora-Communities dar, worüber eine kritische Auseinandersetzung mit den Lebenssituationen von Kurd/inn/en im Residenzland stattfinden kann (SiX-Hohenbalken 2013, p. 15 f.). Wie Studien belegen (Demmers 2007; Burgess 2014) beeinflusst der Grad an In- oder Exklusion in der Aufnahmegesellschaft auch wesentlich das Ausmaß an Herkunftsorientierung in einer Diaspora. Im Fall der Kurd/ inn/en ist bedingt durch religiöse und weltanschauliche Liberalität, Säkularität und sich verändernde Gender Role Models auch ein hohes Ausmaß an Akzeptanz und Inklusion dieser Diaspora in der urbanen Gesellschaft Wiens anzunehmen. Dies unterscheidet die Situation der kurdischen Diaspora ganz erheblich von Communities aus anderen stark muslimisch geprägten Herkunftsstaaten (z.B. Afghanistan, Tschetschenien).

\section{Die folgenden Forschungsfragen sollten beantwortet werden:}

○ Wie werden die Perspektiven, Rahmenbedingungen und (lokalen) Opportunitätsstrukturen der Diaspora Entrepreneurship in den kurdischen Communities in Wien beurteilt?

- Wie sehen die persönlichen Erwartungshaltungen, die Entscheidungsfindung und Motive für die selbständige Erwerbstätigkeit aus? Welche Pläne und Intentionen bezüglich des bestehenden Unternehmens oder weiterer Start-ups bestehen? Nach welchen Kriterien erfolgen die Branchenwahl, unternehmerische und Marketingstrategien, Marktpositionierung und die Finanzierung?

- Welche Unterschiede bestehen zwischen Unternehmer/inne/n der ersten, zweiten und dritten Generation der kurdischen Community in Wien?

- Welche sozialen, wirtschaftlichen und politischen Impulse gehen von kurdischen Selbständigen auf unterschiedlichen räumlichen Ebenen (Bezirke, „Grätzel“) in den Wiener Bezirken aus?

○ Welche räumlichen Bindungen sind bei den Unternehmer/inne/n vorhanden und wie und in welchem Ausmaß sind sie in die Wiener Aufnahmegesellschaft integriert? Welche Unterschiede bestehen zu unselbständig erwerbstätigen Kurdinnen und Kurden?

- Welche Barrieren auf dem Weg zum/-r Unternehmer/in hatten die Befragten zu überwinden? Welche spezifischen Herausforderungen (z.B. kulturelle, religiöse, familiäre und geschlechterrollenbezogene, finanzielle) betrafen vor allem die Frauen?

- Wie bewerten die Befragten ihre Rolle innerhalb der Diaspora und die Relevanz der Entrepreneurship im Hinblick auf ihr eigenes Empowerment innerhalb der 
Diaspora-Community sowie in der städtischen Gesellschaft in Wien generell? Welche Erfahrungen haben sie gemacht? Welchen Beitrag zum Empowerment der kurdischen Diaspora in Wien können und wollen sie leisten? Bestehen Verflechtungen mit politischen Aktivitäten und falls ja, welche?

Die Analysen basieren auf den Resultaten der von uns durchgeführten Expert/inn/ eninterviews und auch den von Dezember 2020 bis Juni 2021 durchgeführten Unternehmer/innenbefragungen. Die Unternehmer und Unternehmerinnen wurden zu ihren Einschätzungen bezüglich der Opportunity Structures, spezifischen Herausforderungen des Unternehmer/in-Seins in Wien, ihren Problemen in der Start-up-Phase und nach der Etablierung ihres Geschäftes, aber auch besonders positiven Aspekten der Selbständigkeit in Wien befragt. Die Befragten decken eine große Bandbreite an Wirtschaftsbranchen ab. Im Sample waren sowohl Einpersonenunternehmen als auch etwas größere betriebliche Einheiten repräsentiert, jedoch keine echten Großunternehmen. Die narrativen Interviews wurden mittels qualitativ inhaltsanalytischer Auswertungsmethoden (vgl. Glaser \& Strauss 2008; Mayring 1991, 2010) analysiert.

Der lokal- und wirtschaftspolitische Kontext, in welchem sich Unternehmen von Migrant/inn/en in Wien entwickeln und der institutionelle Kontext bilden Bestandteile der Opportunity Structures, deren Auswirkungen auf kurdische Unternehmer/innen untersucht wurden. Unsere Analyseansätze basierten auf der Wichtigkeit der individuellen und Gruppenressourcen der Immigrant/inn/en und deren Wechselwirkungen mit den strukturellen Kontextbedingungen des Aufnahmelandes. In diesem Teil wurden dann auch persönliche Entscheidungen sowie die Relevanz sozialer Netzwerke einbezogen.

\section{Die Studie setzte also an folgenden Punkten an:}

○ Dem Fehlen empirisch gesicherten Wissens für Wien über die Diaspora Entrepreneurship von Kurd/inn/en aus der Türkei und vor allem aus Syrien, dem Irak und dem Iran.

- Der generell steigenden Bedeutung von Entrepreneurship für die urbane Ökonomie in Wien sowie für die soziale und strukturelle Integration von Immigrant/inn/en.

- Dem hohen Empowerment-Potenzial selbständiger Erwerbstätigkeit für die Wiener kurdische Community im Allgemeinen und für kurdische Frauen im Speziellen. 